\title{
Fratelli ma non troppo. La chiesa cattolica e gli immigrati in Italia
}

\section{Maurizio Ambrosini ${ }^{1}$}

\section{Introduzione. Tema e obiettivi dell'articolo}

Molti immigrati trovano nelle appartenenze, aggregazioni e pratiche religiose un punto di riferimento importante nella loro esperienza di insediamento in terra straniera (Ambrosini, Naso e Paravati, 2018). Sul versante delle società riceventi, le religioni dei migranti sono invece perlopiù viste come un simbolo visibile di alterità e distanza culturale, soprattutto nell'Europa secolarizzata di oggi (cfr. per es. Soehl, 2017 per il caso francese). All'interno stesso delle religioni storiche, l'accoglienza degli immigrati mette in moto dinamiche variegate. Aspetti come la solidarietà verso lo straniero, il dialogo interreligioso, l'apertura verso i correligionari di origine straniera, articolano un panorama di comportamenti diversificati. Tra gerarchie ecclesiastiche, personale religioso, minoranze impegnate e semplici fedeli, il tema dell'immigrazione suscita passioni e divisioni.

In questo articolo mi propongo di sviluppare un versante poco frequentato della questione, quello dei rapporti tra la chiesa cattolica e gli immigrati cattolici nel nostro paese.

La chiesa cattolica italiana ha una storia secolare di impegno a sostegno dei migranti all'estero. Per questa finalità sono sorte congregazioni religiose apposite tra la fine dell' 800 e gli inizi del '900: il vescovo Scalabrini e madre Francesca Cabrini sono stati i più famosi fondatori di istituti religiosi ancora oggi attivi in molti paesi del mondo. Negli Stati Uniti d'America la presenza di istituzioni cattoliche è stata di grande significato per l'assistenza sociale, il mantenimento dell'identità culturale, il collegamento con la madrepatria, oltre all'accompagnamento pastorale, a favore degli italiani e degli altri immigrati cattolici: riprendendo la formula delle $3 \mathrm{R}$ di Hirschman (2004), la chiesa cattolica è stata per molti immigrati una fonte di rifugio, rispetto e risorse.

Negli ultimi decenni, i flussi migratori sono andati prevalentemente in direzione opposta. L'Italia come è noto è diventata un'importante destinazione per gli immigrati provenienti da molti paesi diversi. Si stima che circa un milione siano almeno nominalmente cattolici, provenienti dall'America Latina, dalla Polonia, dall'ovest dell'Ucraina, dalle Filippine, da vari paesi dell'Africa sub-sahariana (IDOS, 2017). La Chiesa cattolica italiana fin dagli inizi del fenomeno si è inserita efficacemente in questo nuovo paesaggio migratorio, svolgendo varie funzioni a sostegno degli immigrati.

Scopo di questo articolo è di distinguere e approfondire i diversi aspetti del rapporto tra chiesa cattolica e immigrati, a partire dal caso italiano. La tesi che intendo avanzare è che la chiesa cattolica è molto attiva nel produrre servizi alle persone, attività di advocacy, rappresentanza politica agli immigrati stranieri; abbastanza aperta nell'offrire spazi e possibilità di incontro alle comunità degli immigrati cattolici e ai loro ministri di culto; poco propositiva invece, spesso indifferente, nei confronti dell'inclusione degli immigrati nelle proprie strutture e nella pastorale ordinaria. Né gli immigrati cattolici appaiono molto rivendicativi in proposito. Quella che vorrei definire come "cittadinanza ecclesiale" degli immigrati stranieri in Italia resta ancora largamente da costruire.

\footnotetext{
${ }^{1}$ Università degli studi di Milano
} 
L'analisi del caso italiano mi induce a formulare un'osservazione di portata teorica più generale: è possibile identificare diverse dimensioni del rapporto tra istituzione ecclesiastica e immigrati, e queste possono seguire andamenti diversi. Il rapporto tra religioni maggioritarie e correligionari immigrati non è monolitico, ma sfaccettato ed esposto a tensioni, al di là della fratellanza dichiarata. Distinguerò in modo particolare: la dimensione della rappresentanza politica e dell'advocacy; quella della protezione sociale e della fornitura di servizi; quella dell'organizzazione comunitaria; quella dell'inclusione nella vita ecclesiale.

\section{Religioni e migranti}

Hagan ed Ebaugh alcuni anni fa (2003) hanno formulato un'osservazione significativa relativamente ai migration studies nordamericani: nonostante la diversità e l'importanza delle credenze e delle pratiche religiose tra gli immigrati, gli studiosi, sia dell'immigrazione, sia delle religioni, hanno trascurato il ruolo della religione e della spiritualità nei processi delle migrazioni internazionali. Le spiegazioni focalizzate sulle motivazioni economiche delle migrazioni hanno tralasciato i contesti culturali in cui le scelte migratorie vengono assunte e perseguite, tra i quali vanno ricordate le risorse spirituali che le religioni forniscono al momento della decisione di emigrare, e gli effetti psicologici che ne discendono sulla resilienza dei migranti di fronte alle avversità e alle prove a cui sono esposti. Aggiungerei: oltre alle risorse spirituali, le istituzioni religiose hanno fornito ai migranti risorse materiali, sotto forma di assistenza e sostegno nelle difficoltà dei processi di insediamento, e risorse sociali, fungendo da catalizzatori, e non di rado da promotori, di reti di relazioni basate sulla duplice appartenenza, confessionale ed etnica. Il loro apporto è stato dunque rilevante su un duplice versante: quello della salvaguardia, o piuttosto della ricostruzione, dell'identità culturale, e quello dell'inserimento nel nuovo contesto (Warner e Wittner, 1998). Anziché opporsi, i due aspetti si sono intrecciati: molti immigrati sono diventati cittadini della nuova società, in cui hanno scelto di riporre le speranze di una vita migliore, proprio grazie alle risorse che l'adesione religiosa ha fornito loro.

Come già notavano Thomas e Znaniecki (1918-20) nel loro studio sull'immigrazione polacca dei primi del secolo scorso, chiese e organizzazioni a base religiosa (educative, mutualistiche, ricreative, ecc.) hanno formato, per molte ondate di nuovi immigrati, una sorta di camera di compensazione, che consentiva agli immigrati di adattarsi al nuovo contesto di vita senza perdere il rapporto con le loro radici identitarie e con le reti sociali dei connazionali. Forse più di quanto credessero i due grandi studiosi questa funzione si è rivelata duratura e capace di transitare da una generazione all'altra.

Sono quindi rilevanti a più livelli i ruoli svolti dalle religioni (e dalle loro istituzioni) nei confronti dei processi di integrazione degli immigrati, in un rapporto dialettico con le istituzioni politiche e con gli atteggiamenti delle società riceventi (Portes e DeWind, 2004). Anzitutto, le istituzioni religiose possono aiutare i migranti nella fase di preparazione del viaggio'2. Dopo l'arrivo, svolgono una funzione di facilitazione dell'insediamento: sul piano culturale, contrastando gli atteggiamenti xenofobi; sul piano politico, favorendo politiche di inclusione; sul piano sociale, fornendo servizi alle persone, e in modo particolare agli strati più deboli (cfr. Hagan 2008, in modo particolare per quanto riguarda l'assistenza agli immigrati in condizione irregolare) . Quando gli immigrati cominciano ad organizzarsi autonomamente, le istituzioni religiose da essi promosse o frequentate diventano capisaldi della difesa del loro patrimonio culturale e della rielaborazione di un'identità soggettivamente significativa e consapevolmente accettata, in

\footnotetext{
${ }^{2}$ Il punto è peraltro controverso e suscettibile di approfondimenti in rapporto ai diversi contesti. E' vero per l'America Latina, ma non per altre regioni del mondo in cui i cattolici si trovano in minoranza, sperimentando tensioni e ostilità. In genere, le minoranze religiose nella società di origine hanno tassi di emigrazione superiori alla media (così avviene per $i$ cristiani in Medio Oriente e in Asia). Le chiese locali però cercano di contrastare la propensione a emigrare dei propri membri, temendo la perdita delle componenti più giovani e intraprendenti e il conseguente impoverimento delle comunità.
} 
cui elementi importati e rielaborati si mescolano con altri, appresi a contatto con la società ricevente. In questo senso, le istituzioni religiose sono un punto d'appoggio per i processi di "acculturazione selettiva" (Portes e Rumbaut, 2001) che mediano tra contesti di origine e società riceventi, cercando altresì di fornire sostegno all'educazione familiare e di evitare che le seconde generazioni entrino in spirali di esclusione sociale.

In ambito cristiano, le chiese dei migranti hanno di solito spiccate caratteristiche «etniche». Sono luoghi della memoria, della conservazione e trasmissione della lingua e delle tradizioni della madrepatria, della possibilità di esprimersi in modo libero e autentico. Sono luoghi in cui i migranti trovano un rimedio allo sradicamento connesso alla migrazione, alla solitudine, alla povertà delle relazioni, alla fatica della vita quotidiana. Sono per definizione luoghi di incontro, di scambio sociale, di aggregazione in terra straniera. Sono luoghi privilegiati in cui trasmettere alle nuove generazioni non solo i valori religiosi, ma anche tradizioni e appartenenze culturali avvertite come una parte profonda e necessaria di sé, del proprio patrimonio simbolico e identitario (Pace e Ravecca, 2010).

Sono anche luoghi in cui i migranti possono esercitare ruoli di responsabilità e anche di leadership, ottenendo riconoscimenti simbolici spesso negati nella loro esperienza lavorativa e sociale (Warner, 2000). Anche per questo di solito gli immigrati preferiscono ritrovarsi in comunità religiose proprie, organizzate su basi nazionali o linguistiche. Esperienze sociali come la condivisione del cibo, la musica e la festa entrano in modo pervasivo nel calendario e nella trama delle relazioni che si strutturano intorno alle istituzioni religiose.

Le chiese sono quindi inevitabilmente luoghi multi-funzionali, a cui i partecipanti chiedono di fornire risposte a molte esigenze quotidiane, non solo spirituali: socialmente fragili, soltanto parzialmente assistiti da reti parentali indebolite, domandano alle istituzioni religiose consiglio, sostegno e assistenza. Le chiese offrono aiuti concreti, ma consentono anche di costruire reti sociali, di approfondire legami, di produrre e far circolare fiducia interpersonale (Cadge e Ecklund, 2007). Sono inoltre luoghi in cui si strutturano percorsi di integrazione, nel senso che i responsabili delle comunità e le stesse relazioni orizzontali tra i partecipanti instillano abiti mentali, regole di comportamento, esempi di buone pratiche di vita che vanno nella direzione del rispetto delle leggi e dei doveri morali nei confronti dei propri simili, come pure della società di accoglienza (Foner e Alba, 2008). La «cittadinanza religiosa» ha inerenti implicazioni civiche (Levitt, 2008). Il riscatto dell'immagine dei migranti dagli stereotipi che li accompagnano, e soprattutto dai pregiudizi che li colpiscono, passa anche attraverso la promozione di stili di vita irreprensibili, la tensione verso modelli normativi esemplari, l'educazione a virtù personali che diventano capitale sociale e simbolico (Zhou e Bankston, 1996).

Per quanto riguarda in modo particolare la chiesa cattolica, l'acculturazione selettiva degli immigrati è strettamente connessa con l'insediamento di istituzioni che mantengono viva la lingua e la cultura del paese di origine. Le funzioni di costruzione dell'identità culturale, di socializzazione, di mutuo aiuto, sono rafforzate dalla possibilità di ritrovarsi in comunità di connazionali relativamente autonome all'interno dell'arcipelago cattolico. Nella storia sociale nordamericana, questo avveniva tra gli italiani anche per reazione contro un'istituzione ecclesiastica locale dominata dalla componente di origine irlandese. Istituendo parrocchie "nazionali" oltre che territoriali, la chiesa cattolica consentiva di conservare la lingua della madrepatria: nel 1916, circa metà dei cattolici frequentavano chiese in cui il linguaggio usato nelle prediche era diverso dall'inglese (Vecoli, 1969). Contro le pressioni della chiesa statunitense verso un'americanizzazione forzata, che significava di fatto una subordinazione alla prassi religiosa e alla gerarchia ecclesiastica irlandese ("il cattolicesimo di un'altra razza", come si esprimeva un prete italiano dell'epoca), la pastorale dei missionari italiani mirava a costruire un'integrazione nella nuova società che non avesse come presupposto la rimozione (e persino la vergogna) delle proprie radici. Già in quell'esperienza storica si verificava quindi una tensione tra l'appartenenza cattolica e la partecipazione alla chiesa locale: gli immigrati preferivano frequentare le proprie comunità, guidate da religiosi provenienti dalla madrepatria. 
Nell'Europa post-bellica, gli emigranti cattolici hanno seguito il medesimo modello: quello della frequentazione di cappellanie a base nazionale e linguistica.

Infine, una dimensione del fenomeno religioso posta in rilievo negli ultimi anni (Levitt, 2003; Ricucci, 2017) consiste nella costruzione e alimentazione di legami transnazionali, che per i migranti significano soprattutto ponti di collegamento tra i luoghi di origine e quelli di insediamento: un aspetto che innesta la questione delle pratiche religiose degli immigrati nel vivo del dibattito sul transnazionalismo. La chiesa cattolica a questo riguardo ha rappresentato un prototipo, con la sua articolazione tra centralizzazione e capillare ramificazione nei contesti locali, con l'attività delle congregazioni missionarie e con la stessa organizzazione della pastorale dei migranti. Oggi, in varie forme questi legami si espandono e intensificano, nelle diverse istituzioni e denominazioni religiose: fondazione di centri religiosi "affiliati"; partecipazione a movimenti religiosi transnazionali che funzionano come membership card per essere riconosciuti e accolti anche all'estero; visite di leader spirituali provenienti dalla madrepatria e richiesta di ministri di culto per l'assistenza pastorale dei migranti; organizzazione di collette e invii di aiuti verso le comunità religiose di provenienza; pratica dei pellegrinaggi verso luoghi santi oltre i confini: sono questi alcuni degli esempi dei legami transnazionali che le religioni istituiscono nell'esperienza dei migranti.

\section{L'azione della chiesa cattolica per gli immigrati}

L'Italia occupa una posizione peculiare nel panorama internazionale dell'immigrazione e delle politiche migratorie. A livello economico e sociale, è stata per circa 25 anni uno dei paesi più aperti d'Europa: ha promulgato sette sanatorie maggiori, oltre ad altri provvedimenti minori o impliciti (come i decreti-flussi), concedendo uno status di residenti legali a oltre cinque milioni di cittadini stranieri e assorbendone 2,4 milioni nel mercato del lavoro regolare (oltre il 10\% dell'occupazione) (Ministero del lavoro, 2017). A livello politico, ha invece dimostrato una notevole riluttanza ad accettare il suo nuovo status di paese ricevente e la sua trasformazione in senso multietnico (Ambrosini, 2013a). Come è noto, ha modificato la legge sulla cittadinanza nel 1992, in reazione al cambiamento in corso, scegliendo un approccio restrittivo e orientato al passato: mantenimento o agevole recupero della cittadinanza per i discendenti di emigranti italiani, tempi di residenza raddoppiati per gli immigrati extracomunitari (Zincone, 2006). Inoltre, comprende nel suo paesaggio politico uno dei più antichi e forti partiti xenofobi d'Europa, a cui sono state affidate per diversi anni competenze-chiave sulla materia, come il Ministero degli interni sotto l'ultimo governo Berlusconi (2008-2011). Il suo recente successo elettorale (marzo 2018), abbinato con le oscillanti ma sostanziali chiusure sulle questioni dell'immigrazione e dell'asilo da parte del Movimento Cinque Stelle, non fa prevedere grandi avanzamenti sul tema della cittadinanza nel prossimo futuro.

L'arrivo e l'insediamento degli immigrati stranieri è pertanto avvenuto in modo spontaneo, trainato dalla domanda di manodopera del sistema economico e delle famiglie, ma non previsto né regolato istituzionalmente. Il pilastro delle politiche migratorie italiane, come in Spagna e in Grecia, sono stati i provvedimenti di sanatoria già richiamati (Sciortino, 2011). Tra il 2002 e il 2012 oltre un milione di immigrati hanno acquisito uno status di residenti legali quando erano già in Italia, grazie a una regolarizzazione. Le regolarizzazioni inoltre sono configurate istituzionalmente come concessioni dello Stato a un datore di lavoro, che dichiara di aver assunto illegalmente un immigrato. Ciò equivale a dire che la politica ha legalizzato gli immigrati soltanto dopo che questi erano entrati in Italia, avevano trovato un lavoro e convinto il datore di lavoro a regolarizzarli.

In questo scenario la chiesa cattolica ha contribuito notevolmente a colmare il vuoto delle politiche pubbliche e ha fatto sentire la propria voce in difesa degli immigrati quando questi sono stati presi di mira da politiche restrittive e in alcuni casi discriminatorie, come è avvenuto più volte nel corso degli anni, soprattutto sotto $i$ governi di centro-destra. Caritas e Fondazione Migrantes, dipendenti dalla Conferenza episcopale italiana, 
insieme ad altre organizzazioni cattoliche, sono state tra i 22 soggetti promotori dell'iniziativa popolare per una nuova legge sulla cittadinanza ("L'Italia sono anch'io"), più aperta nei confronti degli immigrati e dei loro figli.

Quindi innanzitutto la chiesa cattolica italiano ha operato a livello politico, richiedendo misure di regolarizzazione per gli immigrati in condizione irregolare, protestando contro discorsi e iniziative xenofobe a livello centrale e locale, promuovendo la riforma della legge sulla cittadinanza. Questo attivismo rientra in uno schema più generale. In un paese in cui l'associazionismo degli immigrati è debole e poco incisivo e la grande maggioranza degli immigrati stranieri non beneficia del diritto di voto, i loro interessi sono stati difesi soprattutto da attori sociali istituzionalizzati e ben inseriti nella società italiana, tra i quali la chiesa cattolica e i sindacati dei lavoratori sono i più importanti.

Si può osservare che la chiesa cattolica italiana nella fase ruggente delle politiche securitarie (2008-2011) non ha spinto le sue critiche fino a compromettere i rapporti con i governi a guida Berlusconi. Le ha spesso affidate a figure di secondo piano o a istituzioni collegate (Caritas, Fondazione Migrantes) e nella sua comunicazione pubblica non ha dato ai temi dell'immigrazione e dell'asilo lo stesso risalto che ha invece conferito ai temi familiari e bioetici. Tuttavia ha sostenuto con coerenza i diritti degli immigrati, anche quando l'opinione pubblica appariva schierata in larga maggioranza dalla parte dei governi di centro-destra e delle politiche restrittive, l'opposizione di sinistra esitava ad alzare la voce, molti fedeli mostravano di non seguire i pastori della chiesa sull'argomento. Negli anni più recenti, con l'avvento di papa Francesco, il nuovo corso della CEI e la cosiddetta emergenza rifugiati, il discorso ecclesiastico si è fatto ancora più convinto, e forse anche più distante dalla sensibilità politica della base dei praticanti.

In secondo luogo, la chiesa cattolica italiana, nelle sue ramificate componenti istituzionali e spontaneistiche, è stata sollecita nello sviluppare un'offerta di servizi dedicati agli immigrati (per es., scuole gratuite di lingua italiana presso molte parrocchie e associazioni), sia riconvertendo verso gli immigrati altri servizi già esistenti (per es. i cosiddetti "centri di ascolto", che forniscono in genere aiuti di prima necessità). Ancora oggi, le istituzioni e associazioni in vario modo collegate con la chiesa cattolica forniscono molti servizi agli immigrati, e specialmente alle componenti più deboli della popolazione straniera: cure mediche per gli immigrati in condizione irregolare o con difficoltà di accesso ai servizi pubblici, mense gratuite per le persone in difficoltà, aiuto nello studio e animazione del tempo libero per i minori.

Le domande di regolarizzazione, ricongiungimento familiare, rinnovo dei permessi di soggiorno, sono istruite da servizi allestiti da attori sociali italiani, tra cui spiccano accanto ai sindacati le organizzazioni cattoliche. In un'indagine sui lavoratori immigrati regolarizzati a Milano con la sanatoria del 2002, il 54,9\% degli intervistati aveva dichiarato di aver ricevuto qualche tipo di aiuto da organizzazioni della società civile italiana, per esempio informazioni sulle procedure per mettersi in regola $(23,4 \%)$, pasti gratuiti $(21,7 \%)$, corsi di italiano (12,3\%), alloggio in ricoveri per persone senza dimora (12,3\%) (Ambrosini e Salati 2004).

Nel contesto italiano come in altri paesi soprattutto l'assistenza nelle necessità più immediate è largamente fornita da organizzazioni religiose, generalmente poco propense a differenziare i propri aiuti in funzione della regolarità dello status dei richiedenti, e proprio per questo frequentate in modo particolare dagli immigrati che hanno difficoltà ad accedere ai servizi formali. Per esempio a Milano esiste un'efficace rete di ambulatori che offrono servizi medici gratuiti agli immigrati in condizione irregolare, dove prestano servizio centinaia di medici volontari (Ambrosini, 2015). Quasi tutti questi ambulatori sono legati a istituzioni cattoliche e hanno sede in strutture di proprietà ecclesiastica. In molte parrocchie operano servizi gratuiti di sostegno scolastico per bambini e ragazzi, e oggi questi servizi accolgono in prevalenza alunni di origine immigrata.

Anche questo impegno non è stato esente da critiche: forze politiche e mass media ostili all'immigrazione hanno spesso accusato la chiesa cattolica di privilegiare gli immigrati rispetto agli italiani in condizione di necessità. Persino a volte di aiutare maggiormente gli immigrati di altre religioni rispetto agli immigrati cattolici. Ciononostante, l'aiuto nei confronti degli immigrati è da anni uno dei principali terreni di impegno 
del volontariato cattolico, delle congregazioni religiose e delle istituzioni caritative collegate alla chiesa cattolica.

Questa scelta risponde dunque a valori molto sentiti nel cattolicesimo italiano socialmente impegnato; ma va comunque osservato che essa tende a istituire una separazione tra i servizi di aiuto e la normale attività parrocchiale, tra i bisognosi aiutati e i partecipanti attivi che fanno realmente parte della comunità. Così quei circuiti positivi di inclusione, crescita di rispettabilità, costruzione di legami sociali, promozione della mobilità, che sono associati negli Stati Uniti alla partecipazione religiosa, in Italia appaiono più stentati.

L'identificazione degli immigrati con i bisognosi è un'importante risorsa motivazionale, per le donazioni e per il coinvolgimento dei volontari. Comporta però un problema: non pochi volontari adottano un linguaggio miserabilista quando parlano degli immigrati. Inconsapevolmente, animati da buone intenzioni, scavano un solco tra se stessi, italiani socialmente integrati, perlopiù di classe media, e i beneficiari del loro aiuto, stranieri socialmente marginali e bisognosi di soccorso.

La Chiesa cattolica ha inoltre favorito l'incontro tra domanda e offerta di lavoro, soprattutto nel settore domestico e assistenziale, anche perché le prime ondate di collaboratrici familiari straniere arrivate in Italia provenivano soprattutto da paesi cattolici (Filippine, El Salvador, Capo Verde...) e sono state aiutate da congregazioni religiose che operavano in quei paesi.

Quando devono assumere un'assistente o una collaboratrice familiare, parecchi datori di lavoro italiani nel tempo si sono rivolti a istituzioni religiose, parrocchie o congregazioni, viste da un lato come garanti della moralità e dell'affidabilità delle lavoratrici, dall'altro come testimoni del fatto che l'assunzione di una persona immigrata, sebbene spesso in condizione irregolare, tende a essere vista e giustificata anche come un gesto di aiuto umanitario. Di qui l'importanza tradizionale delle organizzazioni cattoliche nel settore domestico in Italia (Andall, 2000; Sarti, 2011; Gallo e Scrinzi, 2017), anche prima dell'arrivo delle donne immigrate, ma ravvivata nell'ultimo scorcio del secolo scorso dall'invenzione dal basso e dal tumultuoso sviluppo di quello che può essere definito welfare parallelo o invisibile: a base domestica, a costo contenuto, spesso informale o debolmente formalizzato, ma soprattutto gestito e controllato direttamente dalle famiglie datrici di lavoro, in proprio o per conto degli anziani genitori (Ambrosini, 2013b). Si può forse precisare che il ruolo delle istituzioni religiose è stato probabilmente più pronunciato nelle prime fasi di avvio del fenomeno che nel periodo successivo, quando le reti degli immigrati sono diventate più autonome e autopropulsive.

Così racconta la propria esperienza una lavoratrice ucraina, Nadja, intervistata in una ricerca del 2005, arrivata in Italia per il tramite di un'agenzia di Napoli che favoriva l'ingresso di persone con visto turistico:

Sono partita il 25 gennaio e il 28 avevo già trovato lavoro a Napoli. Lavoravo da una signora e mi trovavo bene. Poi lei poverina è morta e così sono rimasta senza lavoro. La sua famiglia mi voleva aiutare ma io sapevo che al Nord lo stipendio era più alto [...]. Così sono venuta al Nord e ho scoperto che c'era una suora a Brescia [...] Mi avevano detto che era meglio se avessi avuto le referenze [...] Così ho chiamato la signora di Napoli e lei è stata troppo gentile, ha chiamato la suora e ha detto che ero stata a lavorare con una persona anziana, che ero stata brava [A.d ${ }^{3}$. 18, ucraina, 36 anni, in Davi 2005, 107].

Pertanto l'anonima suora bresciana agiva all'epoca come broker nel mercato del lavoro domestico, ponendo in collegamento domanda locale e offerta immigrata, ed era così efficace da essere conosciuta a più di 1.000 $\mathrm{km}$. di distanza.

\footnotetext{
${ }^{3}$ A.d. sta per «assistente domiciliare»: come è noto però in Italia si usa comunemente il termine riduttivo di "badante", che non rende giustizia della complessità e della delicatezza dei compiti assistenziali svolti.
} 
I datori di lavoro trovano anch'essi nei servizi delle istituzioni solidaristiche supporto e consulenza per le procedure di regolarizzazione, spesso tutt'altro che agevoli. Racconta un'intervistata:

Andavo là con mio marito (il riferimento è al SAI: Servizio Accoglienza Immigrati della Caritas di Milano), facevamo due o tre domande, mi hanno chiarito le idee, verificavamo, perché certe cose erano un po' così, un po' nebulose, allora magari riferivo, ho saputo così e così, e dopo tiravamo le conclusioni, sì insomma diciamo che sono stata soddisfatta [I 10, in Corrias 2004, 97].

Anche il coinvolgimento nell'incontro tra domanda e offerta di lavoro nel settore domestico e assistenziale comporta un risvolto problematico: i datori di lavoro cattolici tendono a vedersi come benefattori di lavoratrici anch'esse cattoliche, generalmente apprezzate, ma di fatto identificate come persone di umili condizioni, bisognose di aiuto, consiglio, protezione. Di nuovo, una linea di separazione e un senso di superiorità si insinuano nelle relazioni interpersonali.

\section{Separati in chiesa?}

Ravvivando la tradizione dell'assistenza spirituale agli emigranti, la chiesa cattolica concede chiese o altri spazi alle comunità nazionali degli immigrati cattolici (Ambrosini, Bonizzoni e Molli, 2018). Molte oggi dispongono di preti connazionali che le seguono in Italia. Queste chiese sono importanti luoghi d'incontro e di organizzazione di attività sociali in Italia. Un datore di lavoro ha raccontato:

La loro comunità è molto corposa e quindi non tanto si trova bene per l'Italia in sé, ma perché qui ha un sacco di persone del suo paese e quindi si sono ricostruiti probabilmente una piccola Romania a Milano, non lo so... però hanno una cerchia molto ampia, ma anche al tempo stesso molto unita [...] Beh, la comunità penso organizzi anche qualcosa... durante le loro festività religiose, tipo la Pasqua [...] organizzano degli eventi. Infatti, tra l'altro qualche mese fa ci ha chiesto il permesso di andare un week-end a Roma, ed è andata ovviamente, partendo un giorno prima, perché c'era qualcosa di organizzato da loro a Roma [C.m ${ }^{4}$. 22, figlio, 45 anni circa, in Cominelli 2005, 182].

Nelle ricerche condotte in Trentino (Ambrosini e Boccagni, 2007; Boccagni e Ambrosini, 2012), ha trovato conferma sotto vari profili l'importanza dei luoghi di aggregazione legati alla pratica religiosa: come fonte di sostegno e di identificazione personale (ovvero come luogo in cui poter esprimere la propria fede e appartenenza, nonché come tramite di continuità con la vita passata); come ambito di aggregazione e di socialità; a volte, specie in occasione di ricorrenze particolari (feste patriottiche e simili), come luogo di riconoscimento e di partecipazione pubblica. Il nesso tra reti etniche e aggregazione intorno alle istituzioni religiose è espresso molto chiaramente in questo brano di intervista:

Qui a Trento frequento miei connazionali e preferisco quelli che hanno famiglia, che hanno figli... ci siamo aiutati, siamo andati insieme alla Caritas, abbiamo parlato delle nostre difficoltà e dei problemi che ho, anche se non possono aiutarmi. Frequentiamo la chiesa insieme... ci troviamo alla chiesa di... [L., ucraina, 38 anni, in Italia da otto, in Ambrosini e Boccagni 2007, 83].

La seguente testimonianza di un immigrato latinoamericano, intervistato a Genova in una ricerca del 2010, pone in rilievo la sintesi tra sostegno emotivo contro la solitudine, la possibilità di ritrovare un ruolo attivo

\footnotetext{
${ }^{4}$ C.m. sta per care-manager: il congiunto, di solito una figlia o un figlio, che segue l'anziano genitore, gestisce e media $\mathrm{i}$ rapporti con l'assistente domiciliare
} 
(nel caso, suonare in chiesa), gli aiuti materiali. Un complesso di aiuti simile a quello posto in luce da Hirschman (2004) nel caso americano con la formula delle 3 R.

Chi mi ha aiutato di più è stata la chiesa, Suor Clara, perché qui ho conosciuto tanta gente che mi ha aperto il cammino, la strada. Poi suono la chitarra. La chiesa è un grande aiuto per tanti, perché uno arriva senza famiglia, solo, allora è la chiesa che ti accoglie. Qui non trovi l'informazione che trovi al sindacato, però si trova la parte migliore... Se non hai da mangiare prendi il 62 e vai a Coronata (una chiesa di Genova, n.d.r.) a pranzo, prendi l'1 e vai a Prà (altra chiesa genovese, n.d.r.) a cena, se c'è freddo vai in una chiesa e ti danno i vestiti. È la chiesa la parte più importante per noi migranti, perché è quello che ti spinge, che ti tira su... Io non ero chitarrista, qui sono diventato un po' chitarrista, qui mi diverto, tranquillo [Diego, in Abbatecola 2010, 121-122].

Un'altra testimonianza raccolta nell'ambito della medesima ricerca insiste maggiormente sul sostegno psicologico, imperniato sulla chiesa come luogo d'incontro

Là uscivo solo la domenica, ero diventata amica di gente della zona di Recco, loro mi invitavano alla chiesa. Alla fine è stata per me come una terapia. Piangevamo, io e le amiche, siamo tutte mamme, la chiesa ci ha servito molto. C'era una signora sudamericana che ci ascoltava, ci raccontava anche le cose che lei ha vissuto. Per quello io capii che dovevamo portare tutti e tre i figli, altrimenti tornavo io in Perù [Alicia, in Abbatecola 2010, 121].

Per contro però queste "chiese etniche" non sono integrate quasi mai nella vita normale delle parrocchie cattoliche. Gli immigrati cattolici sono raramente inclusi nei consigli pastorali parrocchiani o diocesani, nelle associazioni cattoliche (se non come operatori o volontari addetti a specifici servizi), o negli organismi rappresentativi del laicato cattolico. Nella diocesi di Milano, la più grande d'Italia, il consiglio pastorale diocesano comprende più di cento persone: preti, religiosi e religiose, laici. Solo di recente (2017) ne sono state cooptate due di origine immigrata, su iniziativa dell'Ufficio per la Pastorale dei migranti.

La vita religiosa degli immigrati è dunque separata da quella della maggioranza italiana. Anche l'offerta di servizi agli immigrati è di solito distinta dalle attività proposte ai fedeli italiani. In diverse parrocchie, soprattutto nei centri storici delle città italiane, immigrati cattolici e parrocchiani italiani condividono la medesima chiesa, ma partecipano a messe e attività pastorali diverse: in inglese, spagnolo o altre lingue per gli immigrati. All'ingresso delle chiese, ci sono avvisi e annunci in italiano per la comunità storica, in lingue straniere per gli immigrati, anche dopo molti anni dall'arrivo in Italia. Si può parlare in proposito di "church sharing", ossia di un uso alternato (non davvero condiviso) dello spazio per il culto.

Questa situazione è ben conosciuta e spesso biasimata dai vescovi e dai responsabili pastorali: il discorso ufficiale sostiene che le comunità dovrebbero amalgamarsi, che si dovrebbe superare la separazione tra fedeli nativi e fedeli immigrati (Ricucci 2017). La responsabilità della separazione è però in genere addossata a questi ultimi. Per gli immigrati in effetti potersi ritrovare, parlare la propria lingua, eseguire i propri canti, partecipare a servizi religiosi nello stile della madrepatria, sono degli incentivi che portano nella direzione di mantenere delle comunità separate da quelle maggioritarie. Per alcuni, come ho già ricordato, questo è anche un modo per esercitare funzioni di leadership, per ottenere dei ruoli di prestigio e dei riconoscimenti che la società ricevente non concede loro. Spesso ci si è domandati per esempio se la rigogliosa vita associativa delle comunità filippine, e specialmente delle donne filippine, non sia in una certa misura compensativa della svalutazione sociale derivante dalla loro occupazione come collaboratrici domestiche (Ambrosini, Bonizzoni e Molli, 2018). Come per gli italiani e tanti altri immigrati cattolici di ieri e di oggi, una comunità che tiene insieme religione, lingua madre, legami sociali con i connazionali, offerta di ruoli sociali di un certo prestigio, ha una capacità di attrazione che la partecipazione ad una comunità religiosa del luogo non riesce a esercitare. Soprattutto se in questo secondo caso si tratta di una partecipazione marginale e subalterna.

Nello stesso tempo va però osservato che fedeli e responsabili pastorali della popolazione maggioritaria non fanno granché per integrare gli immigrati nella vita normale delle parrocchie e delle associazioni cattoliche: 
li aiutano, li difendono, concedono loro spazi per incontrarsi, ma non si mescolano con loro. La percezione di distanza sociale prevale sulla comunanza di fede. I migranti di fatto non vengono riconosciuti come portatori di adeguate competenze per essere inclusi negli organismi di partecipazione alla vita ecclesiale, come $\mathrm{i}$ consigli pastorali. Le loro devozioni, feste, processioni e forme cultuali vengono tollerate ma non integrate nella vita pastorale delle comunità autoctone. Sempre più spesso nelle chiese si trovano immagini sacre "importate" dagli immigrati, ma queste piccole innovazioni estetiche non incidono sull'immaginario religioso dei fedeli italiani. Anche i numerosi preti, religiosi e religiose stranieri, provenienti principalmente dal Sud del mondo, sono sempre più richiesti per compensare la riduzione e l'invecchiamento del personale ecclesiastico, ma raramente occupano posizioni di rilievo nelle curie diocesane o nelle congregazioni religiose.

Si può prendere un altro esempio, riferito alla 47a Settimana Sociale dei Cattolici Italiani (Torino 2013), storico raduno della Chiesa cattolica italiana (la prima Settimana sociale si svolse nel lontano 1908), con rappresentanze provenienti da tutte le diocesi del paese. In quell'ambito si è tenuta un'assemblea tematica sull'immigrazione, il cui titolo era: "Il cammino comune con le famiglie immigrate". Hanno partecipato più di 150 persone, ma tra di esse solo due avevano un background migratorio: un prete che svolgeva attività pastorale a Roma e un impiegato di un ufficio diocesano per gli immigrati a Torino. Per due giorni i delegati hanno discusso con passione dei problemi degli immigrati, condannando politiche e discorsi xenofobi, rinnovando la richiesta di una nuova legge sulla cittadinanza, criticando lo sfruttamento degli immigrati nel lavoro per le imprese, nel lavoro di cura per le famiglie, nell'industria del sesso. Alcuni hanno anche rilevato che lo storico titolo dell'evento andrebbe ormai modificato: non più "Settimana Sociale dei cattolici italiani", ma "Settimana Sociale dei cattolici in Italia", includendo anche i cattolici stranieri residenti. Tutto questo però è avvenuto sostanzialmente senza la partecipazione dei diretti interessati.

Quindi gli immigrati cattolici conducono una vita ecclesiale separata a livello parrocchiale, ritrovandosi in alcune chiese che offrono loro spazi e possibilità di organizzarsi in modo autonomo. Riproducono della loro tradizione religiosa devozioni, momenti rituali, feste religiose, a volte anche processioni che si estendono negli spazi pubblici (Ricucci, 2017): rendono locale la pluriformità del cattolicesimo globale contemporaneo. Sono invece finora sostanzialmente esclusi dalle istituzioni di livello intermedio, come i consigli pastorali diocesani, oltre che dai grandi eventi nazionali della chiesa cattolica italiana.

Va riconosciuto che qualcosa si muove. La diocesi di Milano ha avviato sotto il nuovo arcivescovo, mons. Mario Delpini, un "sinodo minore" dedicato alla "chiesa dalle genti", ossia alla presenza dei fedeli di origine immigrata. I lavori sono in corso (primavera 2018) e si può immaginare ne possano scaturire novità interessanti. Il documento preparatorio (Arcidiocesi di Milano 2018) fatica però ancora una volta a conciliare un linguaggio simpatetico e generoso con un'analisi obiettiva della situazione e dei suoi nodi problematici. Si ricorre molto per esempio al concetto di "meticciato", caro al card. Scola. Il meticciato assunto seriamente dovrebbe comportare però non solo una mescolanza fisica e spaziale, un convergere negli stessi luoghi, ma uno scambio che produca nuove sintesi culturali. Non si vedono invece segni tangibili di come la chiesa milanese, la sua prassi pastorale, la sua liturgia, la sua catechesi, la sua elaborazione teologica siano cambiate per effetto dell'incontro con gli immigrati e dell'influenza di questi. Nel testo si propongono esempi come la partecipazione di ragazzi immigrati agli oratori o ai doposcuola: si confonde l'accoglienza e il sostegno con la mescolanza effettiva, che dovrebbe comportare una trasformazione visibile delle parti coinvolte, compresa la componente maggioritaria.

Il documento preparatorio inoltre sorprendentemente sfiora soltanto in un paio di punti la questione cruciale degli spazi e delle forme di partecipazione attiva degli immigrati nella chiesa locale, senza fornire indicazioni al riguardo. Una retorica spirituale colta e benintenzionata, ricca di riferimenti biblici e magisteriali, espunge l'analisi della realtà mondana e dei suoi problemi. 
Il processo è solo agli inizi, la consultazione delle realtà di base potrà arricchire il quadro, qualche scelta forse s'imporrà. Le premesse però nei fatti appaiono poco incisive, più confermative della realtà esistente che orientate all'innovazione.

\section{Conclusioni. Comunità immigrate e chiesa cattolica maggioritaria: diversi livelli di interazione}

In questo articolo ho distinto aspetti diversi delle relazioni tra la chiesa cattolica italiana e gli immigrati. Come abbiamo visto, le istituzioni cattoliche sono attive nella promozione dei diritti degli immigrati, sono direttamente o indirettamente coinvolte nell'erogazione di vari servizi e forme di aiuto, sono disponibili a concedere spazi alle comunità di immigrati cattolici. Autorizzano l'introduzione di feste e devozioni particolari che esprimono la sensibilità religiosa degli immigrati. Non riescono invece a integrarli nella normale attività pastorale, e nella vita ordinaria della chiesa cattolica la maggioranza nativa e le minoranze immigrate rimangono normalmente separate. A livelli istituzionali più alti (istituzioni diocesane, eventi nazionali), raramente gli immigrati stranieri sono coinvolti.

Nella seguente tabella propongo una sintesi dei principali ambiti di interazione tra chiesa cattolica italiana e immigrati.

Tab.1. Forme di interazione tra la chiesa cattolica italiana e gli immigrati cattolici

\begin{tabular}{|c|c|c|}
\hline Campo di attività & $\begin{array}{l}\text { Ruolo della chiesa cattolica } \\
\text { italiana }\end{array}$ & Ruolo degli immigrati \\
\hline Dibattito politico & $\begin{array}{l}\text { Difesa dei diritti degli immigrati, } \\
\text { condanna delle discriminazioni e } \\
\text { dei discorsi xenofobi, domanda di } \\
\text { regolarizzazioni, accoglienza per i } \\
\text { richiedenti asilo e innovazioni } \\
\text { nelle norme sulla cittadinanza }\end{array}$ & $\begin{array}{l}\text { Debole: principalmente, alcune } \\
\text { dimostrazioni locali da parte di } \\
\text { frange politicizzate. } \\
\text { Cooperazione con attori sociali e } \\
\text { politici italiani }\end{array}$ \\
\hline Offerta di aiuto e servizi & $\begin{array}{l}\text { Ampio coinvolgimento in diverse } \\
\text { forme di sostegno, rivolte } \\
\text { principalmente alle componenti } \\
\text { più deboli della popolazione } \\
\text { immigrata: irregolari, disoccupati, } \\
\text { rifugiati, donne con bambini, } \\
\text { donne sottratte alla prostituzione }\end{array}$ & $\begin{array}{l}\text { Alcuni immigrati collaborano } \\
\text { come operatori sociali con } \\
\text { istituzioni cattoliche e cooperative } \\
\text { collegate. Con il tempo, sta } \\
\text { crescendo anche il numero degli } \\
\text { immigrati impegnati come } \\
\text { volontari in ambito locale }\end{array}$ \\
\hline $\begin{array}{l}\text { Incontro tra domanda e offerta di } \\
\text { lavoro }\end{array}$ & $\begin{array}{l}\text { Soprattutto informale e riferito al } \\
\text { settore domestico-assistenziale }\end{array}$ & $\begin{array}{l}\text { Intermediazione tra reti etniche } \mathrm{e} \\
\text { istituzioni cattoliche }\end{array}$ \\
\hline Luoghi d'incontro & $\begin{array}{l}\text { Concessione di chiese (soprattutto } \\
\text { a part-time: church sharing) e } \\
\text { spazi per gruppi ed eventi sociali }\end{array}$ & $\begin{array}{l}\text { Ruolo attivo nell'organizzazione } \\
\text { di "chiese etniche", con le proprie } \\
\text { attività }\end{array}$ \\
\hline Ritualità e devozioni & $\begin{array}{l}\text { Tolleranza, tiepida accoglienza } \\
\text { delle peculiari devozioni degli } \\
\text { immigrati cattolici }\end{array}$ & $\begin{array}{l}\text { Importazione di rituali, devozioni } \\
\text { particolari, feste e processioni } \\
\text { della propria tradizione locale }\end{array}$ \\
\hline $\begin{array}{l}\text { Inclusione attiva nelle comunità } \\
\text { ecclesiali }\end{array}$ & $\begin{array}{l}\text { Separazione, strutture e attività } \\
\text { differenti per i fedeli immigrati e } \\
\text { per gli italiani }\end{array}$ & $\begin{array}{l}\text { Preferenza per gruppi e attività } \\
\text { separate in cui la condivisione } \\
\text { della lingua facilita la } \\
\text { partecipazione ed eventualmente } \\
\text { l'assunzione di ruoli di leadership }\end{array}$ \\
\hline
\end{tabular}


Da questa analisi penso si possano trarre indicazioni più ampie, meritevoli di approfondimenti comparativi. Il caso degli immigrati che arrivano e si insediano in un paese in cui la religione cattolica è storicamente maggioritaria pone questioni diverse da quelle di paesi in cui il cattolicesimo è importato dagli immigrati. Quando si stabiliscono in un paese di tradizione cattolica, gli immigrati incontrano una società stratificata e una chiesa istituzionalmente radicata. Sono o dovrebbero essere tutelati sotto il profilo politico, in coerenza con gli insegnamenti del magistero sociale cattolico, e sono o dovrebbero essere accolti e assistiti nei loro bisogni sociali essenziali. Ma la loro partecipazione attiva alla vita ecclesiale locale è frenata da barriere linguistiche, sociali e culturali che agiscono su entrambi i versanti: da parte degli immigrati, la formazione di comunità autonome risponde a diversi bisogni, di identità culturale, socialità, mutuo aiuto, riconoscimento; da parte della maggioranza nativa, manca interesse e volontà di includere su un piano di parità degli immigrati percepiti come culturalmente diversi e socialmente marginali. A livelli istituzionali più alti, l'esclusione degli immigrati, preti compresi, è ancora più evidente e rigida.

Lo stesso impegno nell'accoglienza e nell'aiuto alimenta la separazione, erige confini sociali invisibili e spesso inconsapevoli: da una parte stanno coloro che aiutano, dall'altra i bisognosi. Da una parte coloro che hanno competenze e risorse, dall'altra coloro che non le hanno. Da una parte gli integrati, dall'altra $\mathrm{i}$ marginali. Da una parte i cattolici nazionali, dall'altra i fedeli immigrati.

Quando si analizzano i rapporti tra gli immigrati e la chiesa cattolica (e forse, le religioni maggioritarie in un determinato territorio), occorre dunque distinguere con cura i diversi ambiti in cui scambi e interazioni possono avere luogo, cogliendo le tensioni e le contraddizioni tra di essi. In sintesi, malgrado l'impegno a favore degli immigrati, l'eguaglianza e la comunione tra fedeli cattolici di diversa provenienza possono rivelarsi un traguardo lontano. In Italia, la "cittadinanza ecclesiale", intesa come partecipazione attiva, consapevole e paritaria dei fedeli immigrati nell'ambito della chiesa cattolica, resta ancora largamente da costruire.

\section{Riferimenti bibliografici}

Abbatecola E. (2010). Essere madri e padri a distanza. In Ambrosini M. e Abbatecola E. (a cura di) Famiglie in movimento. Separazioni, legami, ritrovamenti nelle famiglie migranti. Genova: Il melangolo. 91130.

Ambrosini M. (2015). NGOs and health services for irregular immigrants in Italy: when the protection of human rights challenges the laws. Journal of Immigrant and Refugee Studies, 13, 2: 116-134

Ambrosini M. (2013a) Immigration in Italy: Between Economic Acceptance and Political Rejection. Journal of international migration and integration 14, 1: 175-194

Ambrosini M., (2013b), Immigrazione irregolare e welfare invisibile. Il lavoro di cura attraverso le frontiere. Bologna: Il Mulino.

Ambrosini, M. e Boccagni, P. (2007) Il cuore in patria. Madri migranti e affetti lontani: le famiglie transnazionali in Trentino. Trento: CINFORMI-Centro informativo per l'immigrazione, Provincia di Trento. 
Ambrosini M., Bonizzoni P. e Molli S. (2018). Immigrati cristiani a Milano: esperienze di partecipazione, aggregazione, integrazione sociale. Annali di Scienze Religiose (in pubblicazione).

Ambrosini M., Naso P. e Paravati C. (a cura di) (2018). Il Dio dei migranti. Pluralismo, conflitto, integrazione. Bologna: Il Mulino (in pubblicazione)

Ambrosini M. e Salati M. (a cura di) (2004). Uscendo dall'ombra. Il processo di regolarizzazione degli immigrati e i suoi limiti. Milano: FrancoAngeli.

Andall J. (2000). Gender, Migration and Domestic Service. The Politics of Black Women in Italy. Aldershot: Ashgate.

Arcidiocesi di Milano (2018). Chiesa dalle genti. Responsabilità e prospettive. Linee diocesane per la pastorale. Documento preparatorio. Milano: ITL.

Boccagni P. e Ambrosini, M. (2012) Cercando il benessere nelle migrazioni. L'esperienza delle assistenti familiari straniere in Trentino. Milano: FrancoAngeli.

Cadge W. e Ecklund E.H. (2007). Immigration and religion. Annual Review of Sociology, 33: 359-379.

Cominelli C. (2005). Obbligazioni extracontrattuali: le attese e $i$ comportamenti. In Ambrosini M. e Cominelli C. (a cura di) Un'assistenza senza confini. Welfare 'leggero', famiglie in affanno, aiutanti domiciliari immigrate. Milano: Osservatorio regionale per l'integrazione e la multietnicità, Regione Lombardia. 159-194.

Corrias R. (2004). Tra prestazioni di servizio e legami personali. Rapporti di lavoro e processo di regolarizzazione nel settore domestico-assistenziale. In Ambrosini M. e Salati M. (a cura di). Uscendo dall'ombra. Il processo di regolarizzazione degli immigrati e i suoi limiti. Milano: FrancoAngeli. 78-99.

Davi M. (2005). Donna e migrante. Il genere tra vincolo e risorsa. In Ambrosini M. e Cominelli C. (a cura di) Un'assistenza senza confini. Welfare 'leggero', famiglie in affanno, aiutanti domiciliari immigrate. Milano: Osservatorio regionale per l'integrazione e la multietnicità, Regione Lombardia. 103-123.

Foner N. e Alba R. (2008). Immigrant religion in the US and Western Europe: Bridge or barrier to inclusion? International Migration Review, 42, 2: 360-392.

Gallo E. e Scrinzi F. 2017. Religione, genere e lavoro di cura. Il caso degli uomini migranti in Italia. Religioni e Società, 32, 88: 74-86

Hagan J.M. (2008) Migration Miracle. Faith, Hope and Meaning on the Undocumented Journey. Cambridge: Harvard University Press.

Hagan J.M. e Ebaugh H.R. (2003). Calling upon the sacred: migrants' use of religion in the migration. process. International Migration Review, 37, 4: 1145-1162.

Hirschman C. (2004). The role of religion in the origin and adaptation of immigrant groups in the United States. International Migration Review, 38, 3:1206-1233.

IDOS (2017), Dossier statistico immigrazione 2017. Roma: IDOS.

Levitt P. (2003). «You know, Abraham was really the first immigrant»: religion and transnational migration. International Migration Review, 37, 3: 847-873

Levitt P. (2008). Religion as a path to civic engagement. Ethnic and Racial Studies, 31, 4: 766-791. 
Ministero del lavoro e delle politiche sociali (2017). Settimo Rapporto annuale sul mercato del lavoro degli immigrati - 2017, Italialavoro, Roma. Scaricabile dal sito: www.lavoro.gov.it

Pace E. e Andrea A. (2010). Religioni, genere e generazioni in movimento. Uno sguardo europeo sull'Italia. Mondi migranti, 4, 2: 43-59.

Portes A. e Rumbaut R.G. (2001). Legacies. The story of the immigrant second generation. BerkeleyNew York: University of California Press- Russel Sage Foundation.

Portes A. e DeWind J. (2004). A cross-atlantic dialogue: the progress of research and theory in the study of international migration. Introduction. International Migration Review, 38, 3: 828-851.

Ricucci R. (2017). Diversi dall'Islam. Figli dell'immigrazione e altre fedi. Bologna: Il Mulino.

Sarti R. (a cura di) 2011. Lavoro domestico e di cura: quali diritti?. Roma: Ediesse

Sciortino G. (2011) (a cura di). Regolarizzazioni. Mondi migranti, 5, 2: 25-134.

Soehl T. (2017). Social Reproduction of Religiosity in the Immigrant Context: The Role of Family Transmission and Family Formation - Evidence from France. International Migration Review, 51, 4: 9991030

Thomas W.I. e Znaniecki F. (1958) [1918-20], The Polish Peasant in Europe and America. New York: Dover Publications.

Vecoli R.I. (1969), Prelates and peasants. Italian immigrants and the Catholic church. Journal of Social history, 2, 3: 217-268

Warner R.S. (2000). The new immigrant religion: an update and appraisal. Epicenter, 5, 2: 1-7.

Warner R.S. e Wittner J. G. (a cura di) (1998), Gatherings in Diaspora: Religious Communities and the New Immigration. Philadelphia: Temple University Press.

Zhou M. e Bankston C.L. (1996). The ethnic church, ethnic identification, and the social adjustment of Vietnamese adolescents. Review of Religious Research, 38, 1: 18-37

Zincone G. (a cura di). 2006 Familismo legale. Come (non) diventare italiani. Roma-Bari: Laterza. 